\title{
The Prevalence of Ranitidine-Associated Thrombocytopenia in Children Admitted in Gov. Celestino Gallares Memorial Hospital
}

\author{
Anabella S. Oncog ${ }^{1}$, Sharon Bohos Fernandez ${ }^{2}$ \\ 1 Department of Pediatrics, Gov. Celestino Gallares Memorial Hospital, Janssen Heights, Dampas District, Tagbilaran City, Bohol 6300, \\ Philippines \\ 2 Department of Pediatrics, Gov. Celestino Gallares Memorial Hospital, Janssen Heights, Dampas District, Tagbilaran City, Bohol 6300, \\ Philippines.E-mail: ciaoidoc@yahoo.com \\ *Corresponding Author: Dr. Anabella S. Oncog, Department of Pediatrics, Gov. Celestino Gallares Memorial Hospital, Janssen \\ Heights, Dampas District, Tagbilaran City, Bohol 6300, Philippines. E-mail: abelle salise@yahoo.com, abelle salise@icloud.com
}

Received: August 26, 2019; Accepted: October 03, 2019

\begin{abstract}
Objective: To determine the prevalence of ranitidine-associated thrombocytopenia in children admitted to Gov. Celestino Gallares Memorial Hospital. Methodology: This is a prospective cohort descriptive study that utilized total population sampling. This study included all patients aged 1 month to 14 years admitted to the pediatrics ward of Gov. Celestino Gallares Memorial Hospital who were given ranitidine for any gastrointestinal indication. Excluded from the study were patients with conditions that pose the risk of thrombocytopenia by themselves, patients with conditions that can possibly increase the platelet count, patients who had no repeat $\mathrm{CBC}$ on the third day of hospitalization, patients given less than 9 doses of ranitidine, and patients who were discharged before the third day of hospitalization. The research protocol was approved by the hospital institutional review board, and a waiver of consent was duly filled up. The researcher noted down the hospital number, age and sex of the patient, platelet count on admission, number of ranitidine doses given, and the repeat platelet count on the third day of hospitalization. All data were tabulated. Mean frequencies and percentages were calculated to describe the demographic features and degree of thrombocytopenia of patients prescribed ranitidine. Pearson's correlation and chi-square test for association were used to measure the association between thrombocytopenia and demographic features of patients who were given ranitidine. Significance was set a $p$-value of $<0.05$. All data analyses were generated using Statistical Package for Social Science (SPSS) version 20.0. Results: There was a total of 59 patients who completed 9 doses of ranitidine. Two of the 59 patients who were given ranitidine had thrombocytopenia, comprising 3.39\% of the cases. Majority of the patients were 1 to 4.9 years of age, with an average age of 2 years. One of the two patients who had thrombocytopenia was 4 years old, and the other child was 10 years old. Both children who had thrombocytopenia were males. Majority of the patients who were given ranitidine for 9 doses did not manifest thrombocytopenia. One had mild thrombocytopenia with a platelet count of $102,000 / \mathrm{LL}$, and the other patient had moderate thrombocytopenia with a platelet count of $90,000 / \mathrm{LL}$. Correlation studies between the demographic features and thrombocytopenia in patients who were given ranitidine revealed that there is no significant association between age and gender and thrombocytopenia, with $\mathrm{p}$ values of 0.534 and 0.418 respectively. Conclusion: Ranitidine-associated thrombocytopenia is a rare condition that occurs in non-critical patients regardless of age and gender. Clinicians should be aware of this risk inasmuch as ranitidine is a commonly-used medication in pediatric population.
\end{abstract}

Keywords: Ranitidine, Thrombocytopenia, Pediatrics, Drug-induced immune thrombocytopenia (DIIT).

\section{INTRODUCTION}

Drug-induced immune thrombocytopenia (DIIT) is an unusual disorder that follows exposure to prescription medications, herbal remedies, foods, and nutritional supplements where drug-dependent antibodies produced by affected individuals bind to platelets and cause platelet destruction [1,2]. It has unsurprisingly become a more frequent cause of isolated 
thrombocytopenia, considering the ever-expanding pharmacopeia today [3].

Drug-induced immune thrombocytopenia was first recognized by Vipan more than 140 years ago when he noted the onset of purpura in patients that he treated with quinine [4]. Even today, quinine remains a well-described cause of DIIT $[5,6]$. Hundreds of additional drugs have been implicated since then $[4,7,8]$.

The exact frequency of DIIT is not known particularly because of the difficulty of diagnosing definitively the condition $[5,7]$. Fortunately, DIIT affects only a small proportion of patients exposed to any one of the culprit medications [5].

The overall incidence of DIIT is estimated to be 10 cases per million population per year [5]. Higher incidence is reported in specific patient populations, including $25 \%$ of critically ill patients9, the elderly [10], and patients taking the drug quinine and sulfamethoxazole-trimethoprim [11]. Incidence of druginduced thrombocytopenia in children could not be ascertained primarily because of paucity of published reports and data. A systematic analysis of children with suspected DIIT conducted in 2013 reported that 11 out of 74 individual patients, and 1 group had definite evidence, and 40 patients and 3 groups had probable evidence for an association of the suspected substance with thrombocytopenia [12]. Another study on druginduced-thrombocytopenia that utilized reports of DIIT in the French Pharmaco Vigilance database reported that DIIT in children were mainly post-vaccinal, involving diphtheria-tetanuspoliomyelitis (DTP), influenza, and mumps-measles-rubella (MMR) vaccines [13].

Life-threatening bleeding may occur due to DIIT; thus, rapid identification of a thrombocytopenia-inducing medication cannot be overemphasized. In general, bleeding from DIIT is mild like petechiae, bruising, and epistaxis [2]. But more severe bleeding manifestations may also occur in the form of gastrointestinal or genitourinary mucosal bleeding, or even intracranial or pulmonary hemorrhage $[5,14,15]$. The platelet count may be lower than 50,000/uL, and nadir levels below 20,000/uL were seen in majority of cases16. In some cases, profound thrombocytopenia as low as $1,000 /$ L may be seen [16]. Hence, DIIT should be considered a serious condition prompting immediate attention because there may occur a rapid drop in the platelet count to a level at high risk for spontaneous hemorrhage [2].

As the term 'drug-induced immune thrombocytopenia' implies, immune processes mediate the thrombocytopenia in these cases. Platelets, more than other marrow-derived cell types, appear to be affected by immune-mediated, drug-dependent destruction [17]. The delay in onset of immune-mediated thrombocytopenia ranges from hours to several years $[5,7,16]$. This variability in onset is determined by whether the patient 1) has preexisting antibodies from a prior exposure to the drug, 2) needs to mount anamnestic response because a previously made antibody has fallen below clinically significant levels, or 3) has never before been exposed to the drug and must undergo primary alloimmunization [2].

DIIT that occurs within 24 hours of exposure to the drug has been proposed to be due to one of two mechanisms. First, the patient has been previously exposed to the offending drug, even if undocumented, and that the acute drop represents a response to preexisting alloantibodies. Second, in case of no prior drug exposure, the patient has preexisting 'naturally occurring' antibodies. These naturally occurring antibodies have been proposed by some researchers to have weak affinity for self- antigens but become more avid for platelet membrane antigens in the setting of exposure to a specific drug $[5,17,18,19]$. The latter proposed mechanism has yet to be fully investigated.

Anamnestic responses require on the order of 3 to 10 days. Primary alloimmunization requires at least 2 to 3 weeks [2].

Ranitidine is a histamine type 2 receptor antagonist, otherwise known as $\mathrm{H} 2$-blocker, that is widely used for treatment of acidpeptic disease and heartburn. It is a competitive and reversible inhibitor of the action of histamine at the histamine $\mathrm{H} 2$ receptors on parietal cells in the stomach, thereby inhibiting the normal and meal-stimulated secretion of gastric acid. It was discovered in 1976 and came into commercial use in 1981 [20]. It is on the World Health Organization's List of Essential Medicines, the most effective and safe medicines needed in a health system [21]. It is used for a lot of indications, including, but not limited to relief of heartburn, short-term and maintenance therapy of gastric and duodenal ulcers, Zollinger-Ellison syndrome, gastroesophageal reflux disease, and erosive esophagitis. It has been studied extensively and has been reported to have an excellent safety profile, with no evidence of dose-related toxicity in regimens encompassing an eightfold range of therapeutic doses [22].

Thrombocytopenia due to $\mathrm{H} 2$-receptor antagonist is a rare but known phenomenon. By 2011, there were 29 cases of H2receptor antagonist-induced thrombocytopenia. These cases were a mix of ranitidine, famotidine, and cimetidine-induced thrombocytopenia [23,24]. Majority of these 29 studies were done on cimetidine, 4 studies on famotidine, and 6 studies on ranitidine. Another case report on thrombocytopenia was reported in India [24], making a total of 7 studies. All 7 studies on ranitidine were conducted on adults with treatment duration ranging from 2 to 18 days [24-30]. As such, concurrent risk factors as well as organ dysfunction were found, and could have confounded the analysis for the association between ranitidine and thrombocytopenia.

At least 2 systematic analyses have implicated ranitidine as one of the drugs more frequently associated with drug-induced thrombocytopenia [31,32]. However, majority of the literatures utilized in these systematic analyses had results for adults.

To date, there is still no published data on the association of ranitidine and thrombocytopenia in children. This study attempted to answer this gap in knowledge.

\section{SIGNIFICANCE OF THE STUDY}

This research paper is believed to benefit the following stakeholders:

\section{A. Gov. Celestino Gallares Memorial Hospital}

This research, which has the potential of worldwide application, supports the institution's vision and mission to become a premier research facility in region VII.

B. Department of Pediatric Medicine of Gov. Celestino Gallares Memorial Hospital

This research will provide basis for standard operating procedures in the use of ranitidine, especially in the setting of dengue endemicity in the country.

\section{The Boholano Children}


Inasmuch as this research will provide basis for rational use of ranitidine, the Boholano children will be assured of the best care that the institution can give.

\section{OBJECTIVES OF THE STUDY}

General objective: To determine the prevalence of ranitidineassociated thrombocytopenia in children admitted to Gov. Celestino Gallares Memorial Hospital

\section{Specific objectives:}

1. To determine the number of children admitted to Gov. Celestino Gallares Memorial who were given ranitidine

2. To determine the demographic features of children given ranitidine, as to:
a. Age
b. Gender

3. To determine the number of cases of thrombocytopenia in patients who were given ranitidine

4. To categorize the degree of thrombocytopenia in patients who were given ranitidine, as to:
a. $\quad$ Mild
b. Moderate
c. Severe

5. To determine if there is significant association between thrombocytopenia, ranitidine administration and the demographic features of children

\section{DEFINITION OF TERMS}

1. Thrombocytopenia $=$ a platelet counts less than $140,000 /$ uL

2. Mild thrombocytopenia $=$ a platelet count of 100,000 to $<140,000 /$ LL $^{[32]}$

3. Moderate thrombocytopenia = a platelet count of 50,000 to $<100,000 /$ uL $^{[32]}$

4. Severe thrombocytopenia $=$ a platelet count of $<50,000 / u L$ [32]

\section{MATERIALS AND METHODS}

Research design: This is a prospective cohort descriptive study.

Study population and locale: This study included patients admitted to Gov. Celestino Gallares Memorial Hospital pediatrics ward who were given ranitidine for any gastrointestinal indication.

Sampling technique: This study utilized total population sampling.

\section{Inclusion and exclusion criteria:}

All patients admitted to the pediatric ward of Gov. Celestino Gallares Memorial Hospital, aged 1 month to 14 years, given ranitidine for any gastrointestinal indication, were included in the study.

Excluded from the study were patients with conditions that pose the risk of thrombocytopenia by themselves, including, but not limited to:

1) Dengue fever

2) Immune-mediated thrombocytopenic purpura

3) Sepsis

4) Disseminated intravascular coagulation

5) Critically ill patients admitted to the pediatric intensive care unit (PICU)

6) Aplastic anemia

7) Leukemia

8) Intake/administration of drugs that can cause thrombocytopenia: heparin, chemotherapeutic agents, quinine, valproic acid, furosemide, penicillin, nonsteroidal antiinflammatory drugs, linezolid, sulfonamides

Excluded also from the study were patients with conditions that can possibly increase the platelet count, including but not limited to:

1) Acute blood loss

2) Hemolytic anemia

3) Sepsis

4) Malignancy

5) Chronic inflammatory disorders such as rheumatoid arthritis, Crohn's disease

6) Post-splenectomy

7) Post-blood transfusion

8) Intake/administration of drugs that can cause thrombocytosis: epinephrine, vinca alkaloids, iron, tretinoin

Excluded also from the study were patients who had no repeat CBC on the third day of hospitalization, those given with ranitidine less than 9 doses, and those who were discharged before the third day of hospitalization.

Duration of the study: This study commenced on May 1, 2018 and ended on April 30, 2019.

\section{Data gathering method:}

1. The researchers sought the approval of the research proposal from the hospital Institutional Review Board (IRB). 
2. After IRB approval was obtained, the researchers wrote to the chief of hospital through the IRB to ask permission to access the charts of patients eligible for inclusion in the study.

3. A waiver of informed consent was duly filled up.

4. After all permissions were granted, the researcher gathered the necessary data from the patients' charts in the ward.

a. The researcher checked all admissions daily for patients given intravenous ranitidine.

b. Eligible patients were written in the patients' registry.

c. The researcher noted down the hospital number, patient's age and gender, platelet result on admission, number of ranitidine doses given, and the repeat platelet on the third day of hospitalization.

\section{Limitation of the study:}

Inasmuch as the hospital complies to the Generics Act of 1988 , the author had no control over the brand of Ranitidine that was used in the study. Thus, this study did not consider the brand of Ranitidine supplied by the hospital pharmacy. Any inter-brand eccentricities that may possibly affect the results of the study were beyond the scope of this study.

\section{Statistical analysis:}

All data were properly noted and tabulated. Mean, frequencies and percentages were calculated to describe the demographic features and degree of thrombocytopenia of patients prescribed ranitidine. Pearson's correlation and Chi-square test for association were used to measure the association between thrombocytopenia and demographic features of patients who were given ranitidine. Significance was set at $p$-value of $<0.05$. All data analyses were generated using Statistical Package for Social Science (SPSS) version 20.0.

\section{RESULTS}

There was a total of 59 patients who completed 9 doses of ranitidine. Two of the 59 patients who were given ranitidine had thrombocytopenia, comprising $3.39 \%$ of the cases.

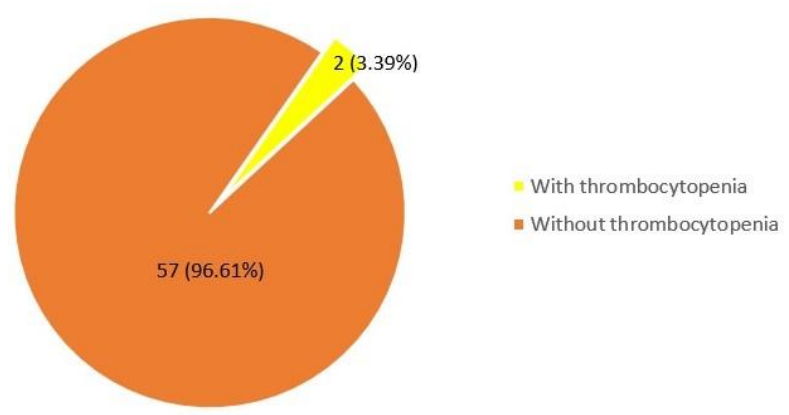

Figure 1: Percentage Distribution of Patients Prescribed Ranitidine with Thrombocytopenia

Majority of the patients were 1 to 4.9 years of age, with an average age of 2 years. One of the two patients who had thrombocytopenia was 4 years old, and the other child was 10 years old.

Table 1: Age of Patients Prescribed Ranitidine

\begin{tabular}{|l|c|c|c|}
\hline \multirow{2}{*}{ Age (years) } & $\mathbf{n}$ & \multicolumn{2}{|c|}{ Thrombocytopenia cases } \\
\cline { 3 - 4 } & & $\mathbf{n}$ & $\%$ \\
\hline $0-0.9$ & 17 & 0 & 0 \\
\hline $1-4.9$ & 34 & 1 & 1.69 \\
\hline $5-9.9$ & 6 & 0 & 0 \\
\hline $10-14.9$ & 3 & 1 & 1.69 \\
\hline Total & $\mathbf{5 9}$ & $\mathbf{2}$ & $\mathbf{3 . 3 9}$ \\
\hline
\end{tabular}

Both children who had thrombocytopenia were males.

Table 2: Gender of Patients Prescribed Ranitidine

\begin{tabular}{|l|c|c|c|}
\hline \multicolumn{1}{|c|}{ Gender } & $\mathbf{n}$ & \multicolumn{2}{c|}{ Thrombocytopenia cases } \\
\cline { 3 - 4 } & & $\mathbf{n}$ & $\%$ \\
\hline Male & 32 & 2 & 3.39 \\
\hline Female & 27 & 0 & 0 \\
\hline Total & $\mathbf{5 9}$ & $\mathbf{2}$ & 3.39 \\
\hline
\end{tabular}

Majority of the patients who were given ranitidine for 9 doses did not manifest thrombocytopenia. One had mild thrombocytopenia with a platelet count of $102,000 / \mathrm{uL}$, and the other patient had moderate thrombocytopenia with a platelet count of 90,000/uL.

Table 3: Degrees of Thrombocytopenia in Patients Prescribed Ranitidine

\begin{tabular}{|l|c|c|}
\hline \multicolumn{1}{|c|}{ Degree of Thrombocytopenia } & $\mathbf{n}$ & $\%$ \\
\hline None & 57 & 96.61 \\
\hline Mild & 1 & 1.69 \\
\hline Moderate & 1 & 1.69 \\
\hline Severe & 0 & 0 \\
\hline Total & $\mathbf{5 9}$ & $\mathbf{1 0 0}$ \\
\hline
\end{tabular}

Correlation studies between the demographic features and thrombocytopenia in patients who were given ranitidine revealed that there is no significant association between age and gender and thrombocytopenia, with $p$ values of 0.534 and 0.418 respectively.

Table 4: Association Between Thrombocytopenia, Ranitidine, and Demographic Features

\begin{tabular}{|c|c|c|c|c|c|}
\hline \multirow{2}{*}{$\begin{array}{c}\text { Demographic } \\
\text { Feature }\end{array}$} & \multicolumn{3}{|c|}{$\begin{array}{c}\text { Degree of } \\
\text { Thrombocytopenia }\end{array}$} & \multirow{2}{*}{$\mathbf{p} \mid$} \\
\cline { 2 - 4 } & Mild & Moderate & Severe & & \\
\hline Age (years) & \multicolumn{3}{|c|}{0.534} & 0.608 \\
\hline $0-0.9$ & 0 & 0 & 0 & & \\
\hline $1-4.9$ & 0 & 1 & 0 & & \\
\hline $5-9.9$ & 0 & 0 & 0 & & \\
\hline $10-14.9$ & 1 & 0 & 0 & & \multirow{2}{*}{0.418} \\
\hline Gender & 1 & 1 & 0 & & \\
\hline Male & 1 & 0 & 0 & & \\
\hline Female & 0 & 0 & & \\
\hline
\end{tabular}


thrombocytopenia.

\section{DISCUSSION}

This study was able to show that the prevalence of thrombocytopenia in patients given ranitidine is 3.39\% and that thrombocytopenia in these children was not associated with neither age nor gender.

Despite extensive literature search, the authors could not find an article on the incidence of thrombocytopenia specifically associated with ranitidine administration in children. There was however a case report of ranitidine-induced thrombocytopenia in a small-for-gestational-age late preterm neonate who showed thrombocytopenia on day 5 of life which was minimally responsive to platelet transfusion, but which recovered completely 5 days after discontinuation of ranitidine [33].

This dearth of information on the association of ranitidine and thrombocytopenia in children supports the statement that druginduced thrombocytopenia caused by $\mathrm{H} 2$-antagonists is a rare phenomenon [33]. Except for the mentioned case above, no other case on drug-induced thrombocytopenia from ranitidine administration in children has been reported. Various case reports on drug-induced thrombocytopenia from $\mathrm{H} 2$-antagonists have been described in adults, mostly from cimetidine, although a few reports were in patients taking ranitidine [24-30]. Furthermore, a systematic review of 74 pediatric drug-induced thrombocytopenia cases that was conducted by Reese et al reported no case of ranitidine or $\mathrm{H} 2$-antagonist as probable or definite cause of the thrombocytopenia [12].

Literatures from adult studies have shown that thrombocytopenia caused by ranitidine is an idiosyncratic reaction associated with an increase in platelet-reactive immunoglobulins and their clearance later by the phagocytic system [24]. The diagnosis is mainly clinical because demonstration of drug-associated antibodies is usually unsatisfactory [34].

\section{CONCLUSION}

Ranitidine-associated thrombocytopenia is a rare condition that occurs in non-critical patients regardless of age and gender. Clinicians should be aware of this risk inasmuch as ranitidine is a commonly used medication in pediatric population.

\section{Recommendations}

To strengthen the association between ranitidine and thrombocytopenia, a causality assessment of the relationship between ranitidine and thrombocytopenia is suggested. In our institution where analysis for drug-dependent platelet-reactive antibodies could not be performed, the WHO-UMC system for standardized case causality assessment may be used.

Corollary to this, a randomized double-blind controlled trial may be conducted to show whether or not there is a significant cause and effect between ranitidine administration and
The author also recommends that ranitidine be not used in patients with dengue to avoid exacerbating the thrombocytopenia in these patients.

\section{Conflict of Interest}

We declare that we have no conflict of interest.

\section{Financial Support}

None declared.

\section{REFERENCES}

1. Aster, R. H. (2007). Drug-induced thrombocytopenia (2nd ed.). In A. D. Michelson (Ed.), Platelets (pp. 887-902). San Diego: Academic Press.

2. Kenney, B., \& Stack, G. (2009). Drug-induced thrombocytopenia. Arch Pathol Lab Med, 133, 309-314.

3. Visentin, G. P., \& Liu, C. Y. (2007). Drug-induced thrombocytopenia. Hematol Oncol Clin North Am, 21, 685-696, vi.

4. Vipan, W. H. (1865). Quinine as a cause of purpura. Lancet, 2, 37.

5. Aster, R. H., \& Bougie, D. W. (2007). Drug-induced thrombocytopenia. N Engl J Med, 357, 580-587.

6. Bougie, D. W., Wilker, P. R., \& Aster, R. H. (2006). Patients with quinine-induced immune thrombocytopenia have both "drugdependent" and "drug-specific" antibodies. Blood, 108, 922-927.

7. George, J. N., Raskob, G. E., Shah, S. R., Rizvi, M. A., Hamilton, S. A., Osborne, S., \& Vondracek, T. (1998). Drug-induced thrombocytopenia: a systematic review of published case reports. Ann Intern Med, 129, 886-890.

8. Rizvi, M. A., Kojouri, K., \& George, J. N. (2001). Drug-induced thrombocytopenia: an updated systematic review. Ann Intern Med, 134, 346.

9. Visentin, G. P., \& Liu, C. Y. (2007). Drug-induced thrombocytopenia. Hematol Oncol Clin North Am, 21, 685-696, vi.

10. Van den Bemt, P. M., Meyboom, R. H., \& Egberts, A. C. (2004). Drug-induced thrombocytopenia. Drug Saf, 27, 1243 - 1252.

11. Kaufman, D. W., Kelly, J. P., Johannes, C. B., Sandler, A., Harmon, D., Stolley, P. D., \& Shapiro, S. (1993). Acute thrombocytopenic purpura in relation to the use of drugs. Blood, 82, 2714-2718.

12. Reese, J. A., Nguyen, L. P., Buchanan, G. R., Terrel, D. R., Vesely, S. K., \& George, J. N. (2013). Drug-induced thrombocytopenia in children. Pediatr Blood Cancer, 60(12), 1975-1981.

13. Moulis, G., Sommet, A., Sailler, L., Lapeyre-Mestre, M., \& Montastruc, J. L. (2012). Drug-induced immune thrombocytopenia: a descriptive survey in the French PharmacoVigilance database. Platelets, 23(6), 490-494.

14. Patnode, N. M., Gandhi, P. J. (2000). Drug-induced thrombocytopenia in the coronary care unit. J Thromb Thrombolysis, 10, 155-167.

15. Von Drygalski, A., Curtis, B. R., Bougie, D. W., McFarland, J. G., Ahl, S., Limbu, I., ..., \& Aster, R. H. (2007). Vancomycin-induced immune thrombocytopenia. N Engl J Med, 356, 904-910.

16. Greinacher, A., Eichler, P., Lubenow, N., \& Kiefel, V. (2001). Druginduced and drug-dependent immune thrombocytopenias. Rev Clin Exp Hematol, 5(3), 166-200.

17. Aster, R. H. (2005). Drug-induced immune cytopenias. Toxicology, 209(2), 149-153.

18. Rizvi, M. A., Shah, S. R., Raskob, G. E., \& George, J. N. (1999). Drug-induced thrombocytopenia. Curr Opin Hematol, 6, 349-353. 
19. Pillai, S. (2005). Two lymphoid roads diverge: but does antigen bade $B$ cells to take the road less traveled? Immunity, 23, 242244.

20. Fischer, J., \& Ganellin, C. R. (Eds.). (2006). Analog-based drug discovery. Weinheim, Germany:WILEY-VCH Verlag GmbH \& Co. KGaA.

21. World Health Organization. (2017). 20th WHO model list of essential medicines. Retrieved from http://www.who.int/medicines/publications/essentialmedicines/en/

22. Mill, J. G., Koch, K. M., Webster, C., Sirgo, M. A., Fitzgerald, K., \& Wood, J. R. (1997). The safety of ranitidine in over a decade of use. Aliment Pharmacol Ther, 11(1), 129-137.

23. Wade, E. E., Rebuck, J. A., Healey, M. A., \& Rogers, F. B. (2002). $\mathrm{H} 2$ antagonist-induced thrombocytopenia: is this a real phenomenon? Intensive Care Med, 28, 459-465.

24. Bangia, A. V., Kamath, N., \& Mohan, V. (2011). Ranitidine-induced thrombocytopenia: a rare drug reaction. Indian J Pharmacol, 43(1), 76-77.

25. Spychal, R. T., \& Wickham, N. W. R. (1985). Thrombocytopenia associated with ranitidine. BMJ, 291, 1687.

26. Pearson, M. W. (1986). Thrombocytopenia associated with ranitidine. BMJ, 292,489.

27. Gibson, P. R., \& Pidcock, M. E. (1986). Immune-mediated thrombocytopenia associated with ranitidine therapy. Med J Aust, $145,661-662$.

28. Gafter, U., Komlos, L., Weinstein, T., Zevin, D., \& Levi, J. (1987). Thrombocytopenia, eosinophilia, and ranitidine. Ann Intern Med, 106,477 .

29. Gafter, U., Zevin, D., Komlos, L., Livni, E., \& Levi, J. (1989). Thrombocytopenia associated with hypersensitivity to ranitidine: possible cross-reactivity with cimetidine. Am J Gastroenterol, 84, 560-562.

30. Bader, A., Carrigan, T., Gopalswamy, N., \& Trulzsch, D. V. (1989). Reversible hematologic suppression during ranitidine treatment. Drug Intell Clin Pharm, 23, 508-509.

31. Reese, J. A., Li, X., Hauben, M., Aster, R. H., Bougie, D. W., Curtis, B. R., ..., \& Vesely, S. K. (2010). Identifying drugs that cause acute thrombocytopenia: an analysis using 3 distinct methods. Blood, 116, 2127-2133.

32. Garbe, E., Andersohn, F., Bronder, E., Salama, A., Klimpel, A., Thomae, M., ..., \& Kurtal, H. (2012). Drug-induced immune thrombocytopenia: results from the Berlin Case-Control Surveillance Study. Eur J Clin Pharmacol, 68(5), 821-832.

33. Hoste, L., \& George, I. (2019). Ranitidine-induced thrombocytopenia in a neonate - a case report and review of literature. J Pediatr Pharmacol Ther, 24(1), 66-71.

34. Caz, P. D., Terrin, J., Dela Rubia, J., Codnna, J., Safont J., \& Mirabet, V. (1995). Ranitidine-induced thrombocytopenia in severe burn patients - a propos of one case. Ann Medit Burns Club. 